$$
\text { CONF- } 9506186--3
$$

\title{
Theoretical Predictions for Exotic Hadrons
}

\author{
T.Barnes \\ Computational and Theoretical Physics Group, Oak Ridge National Laboratory \\ Oak Ridge, TN 37831-6373, USA \\ and \\ Department of Physics and Astronomy, University of Tennessee \\ Knoxville, TN 37996-1200, USA

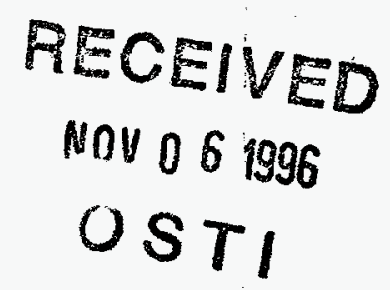

\begin{abstract}
In this contribution we discuss current theoretical expectations for the properties of light meson "exotica", which are meson resonances outside the $q \bar{q}$ quark model. Specifically we discuss expectations for gluonic hadrons (glueballs and hybrids) and muitiquark systems (molecules). Experimental candidates for these states are summarized, and the relevance of a TCF to these studies is stressed.
\end{abstract}

\section{INTRODUCTION}

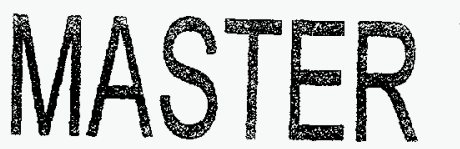

The most exciting developments in QCD spectroscopy involve searches for resonances which are external to the conventional $q \vec{q}$ quark model of mesons. There are two general classes of such states, which are those with dominant gluonic excitations "gluonic hadrons" and states with more quarks and antiquarks than the familiar $q \vec{q}$ states.

Since QCD is a theory which contains both quarks and gluons as dynamical degrees of freedom, we would expect to see evidence of both these building blocks in the spectrum of physical color-singlet hadrons. It is remarkable, however, that of the hundreds of hadronic states now known, most can be described as states made only of quarks and antiquarks in the nonrelativistic quark model, and none of the remaining problematic resonances have been established as having dominant gluonic valence components. The best evidence for the presence of gluons at low energies is indirect, for example in the BreitFermi one-gluon-exchange Hamiltonian used in potential models and in the $q \bar{q} \leftrightarrow s \vec{s}$ configuration mixing evident in the $\eta$ and $\eta^{\prime}$.

In addition to these gluonic states, one may also form color singlet combinations from multiquark systems of quarks and antiquarks, beginning with $q^{2} \bar{q}^{2}$.

\section{DISTRIBUTION OF THIS DOCUMENT IS UNUMITED}




\section{DISCLAIMER}

This report was prepared as an account of work sponsored by an agency of the United States Government. Neither the United States Government nor any agency thereof, nor any of their employees, makes any warranty, express or implied, or assumes any legal liability or responsibility for the accuracy, completeness, or usefulness of any information, apparatus, product, or process disclosed, or represents that its use would not infringe privately owned rights. Reference herein to any specific commercial product, process, or service by trade name, trademark, manufacturer, or otherwise does not necessarily constitute or imply its endorsement, recommendation, or favoring by the United States Government or any agency thereof. The views and opinions of authors expressed herein do not necessarily state or reflect those of the United States Government or any agency thereof. 


\section{DISCLAMMER}

Portions of this document may be illegible in electronic image products. Images are produced from the best available original document. 
Although these have been quite controversial, it now appears that light multiquark resonances do exist in nature, albeit as bound meson pairs "molecules" rather than single four-quark clusters.

Experimental studies now in progress may alter the status of hadronic exotica considerably, since there are now several resonances that, if confirmed, appear to be likely candidates for glueballs, hybrids and additional molecules. As we shall see, these states share several common features with theoretical expectations for these unusual hadronic states.

In this contribution we will review current theoretical expectations for gluonic hadrons and molecules, and briefly discuss some of the experimental candidates for these states.

\section{GLUEBALLS}

\section{A. Introduction}

A priori one would expect glueballs to be the most attractive gluonic hadrons experimentally, since they might be expected to differ most noticeably from $q \bar{q}$. In practice this naive expectation may not be realized; studies of the light glueball spectrum using lattice gauge theory have found that the lowool-lylug gluoball in achlar, and tis coupllis to two-panudesncalar final states suggests a typical hadronic width. The next glueballs encountered at higher masses are predicted to be $0^{-+}$and $2^{++}$, and states which couple to two transverse gluons (presurnably the lightest glueballs) do not contain exotic $J^{P C}$.

Although there have been many studies of the spectrum and quantum numbers expected for glueballs [1], the results of lattice gauge theory should be treated as the most relevant to experiment, since they bear the closest resemblance to full QCD. The assumptions of quenched lattice gauge theory are that decay channels do not modify glueball masses significantly (since the neglect of quarks implies stable light glueballs) and that the extrapolations to small lattice spacing and large lattice volume do not introduce important biases. If glueballs are not very broad objects, the assumption of stable glueballs should not introduce large mass errors.

There are lattice predictions for the masses of glueballs with various $J^{P C}$ [2]; the most reliable is presumably for the scalar glueball ground state, which is predicted to have a mass of

$$
M\left(0^{++}\right)= \begin{cases}1.550(50) & \mathrm{GeV}[3] \\ 1.740(71) & \mathrm{GeV}[4]\end{cases}
$$

The corresponding mass estimate for the tensor glueballs is in the $2.2-2.4 \mathrm{GeV}$ range,

$$
M\left(2^{++}\right)= \begin{cases}2.270(100) & \mathrm{GeV}[3] \\ 2.359(128) & \mathrm{GeV}[4] ;\end{cases}
$$

with the pseudoscalar glueball at a similar mass.

There are obvious problems associated with the identification of a scalia state near $1.5 \mathrm{GeV}$. The $f_{0}$ sector is the most complicated of all meson sectors. with at least six problematical states, $f_{0}(980), f_{0}(1300), f_{0}(1365), f_{0}(1501)$ $f_{0}(1590)$ and $f_{0}(1710)$. Since this sector contains broad and overlapping reso nances, the problem of identifying unusual states against the $q \vec{q}$ and $s \bar{s}$ back ground, and the related problems of separating individual resonances from interference and threshold effects are daunting ones. If the scalar gluchall does have a typical harlronic width, as suggested by the work of Sexton ef al [5], it may be quite difficult to identify this state convincingly. Amsler and Close [6] note that the near degeneracy of the pure (quenched) LC'I gluebal and the $L=1 q \bar{q}$ and $s \vec{s}$ multiplets may lead to complicated mixing elfects, si the physical states may be nontrivial combinations in flavor space, as in the $\eta-\eta^{\prime}$ sector.

The tensor glueball may be an easier experimental target, since 1.ho pected mass is fat above the lowest-lying $2^{++}$quarkonium stales. Hore the problem is that the mass region above $2 \mathrm{GeV}$ is poorly explored, so it is nol yet posslblo to distingulsh a tensor glucball from the linckeronind of melial ${ }^{3} P_{2}$ and ${ }^{3} F_{2} q \bar{q}$ and $s \bar{s}$ stales. This lack of adequate information regarding the higher mass quarkonium spectrum is even more of a problem in the $0^{-+}$ sector.

\section{B. Expectations for glueball properties}

Since we have no confirmed glueballs and the states predicted are in chan nels with a complicated or poorly explored resonance spectrum, it would lie useful to have reliable theoretical predictions of glucball properties as a guicte. The data we are likely to have on gluonic candidaten in the nenr future nr. their masses, widths and strong decay amplitudes. Here a very charactoristir. naive glueball signature can be given, although it is easy to imagine ways in which this signature might be violated.

As gluons at the bare lagrangian level have equal strength couplings in quarks of all flavors, one can make the assumption that flavor-symmetric con plings to hadron final states are approximately valid for physical glueballs. This gives a characteristic flavor-singlet branching fraction to pseudoscalar pairs, which is (neglecting phase space differences)

$\Gamma\left(G \rightarrow \pi \pi: K \widetilde{K}: \eta \eta: \eta \eta^{\prime}: \eta^{\prime} \eta^{\prime}\right) /($ phase space $)=3: 4: 1: 0: 1$. (3)

Of course this simple pattern should at least incorporate the $|\vec{p}|$ from phase space for an S-wave decay, and there is in addition a decay form factor 
which depends on the unknown scalar glueball wavefunction and the decay mechanism. Experience with the ${ }^{3} P_{0}$-model $f_{0}(q \vec{q})$ decay amplitude to $\pi \pi$, which has a node near the physical point [7], suggests that the naive patiern of flavor-singlet decay amplitudes may indeed be far from the physical couplings.

The accuracy of naive flavor-singlet couplings can be tested for a pure (quenched) scalar glueball in lattice gauge theory through a determination of the glucball-Ps-Ps three point function. Preliminary results for this coupling [5] indicate that flavor-singlet symmetry may indeed be badly violated at the amplitude level, and higher-mass Ps pairs are preferred in the decay. In view of the relatively large errors it is important to improve the statistics of this interesting lattice gange theory measurement. An extension of this work to the decay amplitudes of tensor and pseudoscalar glueballs would also be a very useful contribution.

In future experimental work it may be possible to determine or limit elec tromagnetic couplings of glueball candidates. Measurements of one-photon $(R \rightarrow \gamma q \bar{q})$ and two-photon $(R \rightarrow \gamma \gamma)$ transition rates of these resonances are extrendy important because theoriats can calculate these for $q \dot{\vec{q}}$ stwhes with reasonably accuracy [8]. The radiative transition rates of a relatively pure glueball would clearly be anomalous relative to expectations for the corresponding $f_{J}(q \vec{q})$ state. If physical glueballs are indeed strongly mixed linear combinations of gluonic, $q \bar{q}$ and $s \bar{s}$ basis states, a convincing way to identify the flavor components of these mixed states would be through a comparison of the relative rates

$$
\mathrm{I}\left(R \rightarrow \gamma \rho^{0}: \gamma \omega: \gamma \phi\right)
$$

since these act as flavor tags. Similarly, $\gamma \gamma$ couplings can be used to locate the scalar nonstrange $f_{0} q \bar{q}$ signal, since this state should have a strong coupling to $\gamma \gamma$. Results on this reaction have already been obtained by the Crystal Ball in the reaction $\gamma \gamma \rightarrow \pi^{\circ} \pi^{\circ}[9]$. Since a glucball should have suppressed couplings to $\gamma \gamma$, measurements of the $\gamma \gamma$ couplings of the various $f_{d}$ states and other light resonances would be very important contributions to light meson spectroscopy at a TCF.

\section{Summary of glueball candidates}

At present the two most prominent experimental candidates for gheballs are the scalar $f_{0}(1500)$ and the $\xi(2230)$, which is probably a tensor. The scalar candidate has a mass and width (as reported by Crystal Barrel [10]) of

$$
M\left(f_{0}\right)=1520+20 \mathrm{MeV}
$$

a.nol

$$
\Gamma\left(f_{0}\right)=148 \underset{-25}{+20} \mathrm{MeV} .
$$

The $f_{0}(1500)$ seems rather too massive to be a nonstrange ${ }^{3} P_{0} q \bar{q}$ stale, but is consistent with the lower mass estimates from IGT for a scalar glueball. 'Tlic width is also quite narrow for a ${ }^{3} P_{0} q \bar{q}$ state at this mass. The decay pattern to pseudoscalar pairs is however inconsistent with flavor symmetry; the squared invariant couplings cited by Amsler [10] are

$$
\begin{gathered}
\Gamma\left(f_{0}(1500) \rightarrow \pi \pi: K \bar{K}: \eta \eta: \eta \eta^{\prime}\right) /(\text { p.s. })= \\
1:<1 / 8.6(95 \% \text { c.l. }): 0.24 \pm 0.12: 0.35 \pm 0.15
\end{gathered}
$$

A priori this argues against a pure glueball interpretation, and subseculeut. work by Amsler and Close [6] has investigated the possibility that these drcays may be consistent with a scalar glueball that has important $q \vec{q}$ and $s .4$

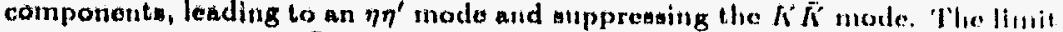
on the coupling to $K \hat{K}$ is actually inferred from another exporiment, and a more careful study of this coupling including interferences at the Crystal Barrel appears to find a much larger $K \bar{K}$ coupling [11]. This state has also berm reported in a recent reanalysis of the MarkIII data on $\psi \rightarrow \gamma \pi^{+} \pi^{-} \pi^{+} \pi^{-}$hy Bugg et al. [12]; in this channel the $f_{0}(1500)$ appears dominantly in the " $\sigma \sigma$ " mode of two $\mathrm{S}$-wave $\pi \pi$ pairs.

The second glueball candidate, which might be the $\xi(22: 30)$ previously wported by MarkIII [13] in $\psi$ radiative decays, is reported by BLS [1.1] to hatse very anomalous properties for a tensor above $2 \mathrm{GeV}$. 'The mass and width $33 \mathrm{l}$. cite for this state in $K_{S} K_{S}$ are

$$
M(\xi)=2232 \underset{-7}{+8} \pm 15 \mathrm{M} \cdot \mathrm{V}
$$

and

$$
\Gamma(\xi)=20_{-16}^{+25} \pm 10 \mathrm{MeV}
$$

with similar results in $P \vec{P}, K^{+} K^{-}$and $\pi^{+} \pi^{-}$. If this narrow state is conlimmerl it is a remarkable discovery indeed. The mass is consistent with licl' expere tations for the lightest tensor glueball (2), and the narrow width implies that this is certainly not a tensor quarkonium state. Since the couplings $10 \pi \pi \mathrm{and}$ $K \bar{K}$ appear to be approximately flavor symmetric [1.1], this appears to be at natural glueball candidate.

Although Godfrey, Kokoski and Isgur [15] previously suggested that the ${ }^{3} F_{2}$ and ${ }^{3} F_{4}$ ss states expected near this mass could be relatively narmw. subsecutent work by Blundell and Codfrey [16] has shown that other mosks 
such as $K_{1}(1270) K$ are large, so $\Gamma\left(f_{2}(s \bar{s})\right) \geq 400 \mathrm{MeV}$. Similarly for the ${ }^{3} F_{4}$ Blundell and Godfrey now find a broader state given these additional modes, $\Gamma\left(f_{4}(s \bar{s})\right) \geq 130 \mathrm{MeV}$. Thus the $s \bar{s}$ assignments now appear implausible if the $\xi(2230)$ does indeed have an experimental width of $<50 \mathrm{MeV}$.

Several of the properties reported for this narrow $\xi(2230)$ are disturbing. It has surprisingly small branching fractions to pseudoscalar pairs in view of the available phase space [14]; branching fractions of only a few percent are implied by the PS185 limit on $P \bar{P} \rightarrow \xi \rightarrow K \bar{K}$. A more important concern is that the reported statistical significance in each of the four channels studied by BES is rather small, $\approx 3 \sigma$. A caution is appropriate because some previously reported narrow effects were subsequently found to be artifacts (for example the $\zeta(8.3)$ ). In view of the remarkable properties reported for this state, measurement of these channels with higher statistics is an extremely important task for any $e^{+} e^{-}$facility operating at the $\psi$ mass.

Although we have only discussed the $f_{0}(1500)$ and $\xi(2230)$ glueball candidates, this is largely because they have attracted considerable attention recently. Several other states with similar masses and the same quantum num bers, notably the $f_{0}(1710)$, should also be considered glueball candidates [5] Measurements of strong branching fractions and electromagnetic decays of this and other glueball candidates should be considered high priorities at a TCF.

\section{HYBRIDS}

\section{A. Introduction}

Hybrid mesons may be defined as resonances in which the dominant valence basis state is $q \bar{q}$ combined with a gluonic excitation. Hybrids are attractive experimentally because, unlike glueballs, they span complete flavor nonets and hence provide many possibilities for experimental detection. In addition, the lightest hybrid multiplet is expected to include at least one $J^{P C}$-exotic (forbidden to $q \bar{q}$ ). In the bag model, for example, the lightest gluon mode has $J^{P}=1^{+}$, so the lowest-lying $q \bar{q} g$ multiplet contains the quantum numbers

$$
J^{P C_{n}}(q \bar{q} g)=\left\{\begin{array}{ll}
0^{-+}, 1^{-+}, 2^{-+} & \left(S_{q \bar{q}}=1\right), \\
1^{--} & \left(S_{q \bar{q}}=0\right) .
\end{array} .\right.
$$

The flux tube model extends this bag model list by adding a degenerate set with reversed $\{P, C\}$ to the lowest hybrid multiplet. Constituent gluon models differ in that their lowest hybrid multiplet has P-wave $q \bar{q}$ quantum numbers [17] and so is nonexotic, although exotics appear in excited hybrid multiplets. An investigation of $q \bar{q} g$ interpolating fields [18] shows that hybrids can have any $J^{P C}$.

\section{B. Hybrid masses.}

Hybrids have been studied using a wide range of models and techniques. These are the MIT bag model [19], constituent gluon models $[17,20,21\}$, the flux tube model [22-31], an adiabatic heavy-quark bag model [32], heavy-quark lattice gauge theory [33] and QCD sum rules [34-38]. There have been no published Monte Carlo lattice gauge theory studies of hybrid masses; a study of exotic hybrid masses would be an interesting application of this technique. In all the theoretical approaches employed to date the lightest hybrids $\left(H_{\eta}\right.$, involving $u, d$ flavors) are predicted to have masses in the $\approx 1 \frac{1}{2}-2 \mathrm{GeV}$ region. A summary of hybrid mass predictions for the especially interesting $1^{-+}$exoti is given in the table below, taken from [28]. A more detailed discussion of these predictions and the literature on hybrids is given by Barnes, Close ancl Swanson [28]; for other recent reviews of hybrids see [39].

Much of the recent interest in hybrids has derived from the flux tube model. which gives rather precise predictions for masses and decay modes of hybrids. The original flux tube references [23-25] cited masses of $\approx 1.9 \mathrm{GeV}$ for the lightest $(u, d)$ hybrid multiplet, $\approx 4.3 \mathrm{GeV}$ for cê hybrids and $\approx 10.8$ (ieV for $b \bar{b}$ hybrids. There is an overall variation of about $0.2-0.3 \mathrm{GeV}$ in these predictions, as indicated in Table 1. Multiplet splittings are usually neglected in the flux tube model. This approximation may not be justified; a large inverted spin-orbit term was found for hybrids by Merlin and Paton [25].

TABLE I. Predicted $1^{-+}$Ilybrid Masses.

\begin{tabular}{llll}
\hline \hline state & mass $(\mathrm{GeV})$ & model & Ref. \\
\hline$H_{\mathrm{u}, d}$ & $1.3 \cdot 1.8$ & bag model & {$[19]$} \\
& $1.8-2.0$ & flux tube model & {$[22-25.28]$} \\
& $2.1 \cdot 2.5$ & QCD sum rules (most after 1984) & {$[35 \cdot 37]$} \\
& 2.1 & constituent gluon model & {$[21]$} \\
\hline$H_{c}$ & $\approx 3.9$ & adiabatic bag model & {$[32]$} \\
& $4.1-4.5$ & flux tube model & {$[23-25,28]$} \\
& $4.1-5.3$ & QCD sum rules (most after 1984) & {$[35-37]$} \\
& $4.19(3) \pm$ sys. & HQLGT & {$[33]$} \\
\hline$H_{b}$ & $10.49(20)$ & adiabatic bag model & {$[32]$} \\
& $10.8-11.1$ & flux tube model & {$[23 \cdot 25]$} \\
& $10.6-11.2$ & QCD sum rules (most after 1984) & {$[35,37]$} \\
& $10.81(3) \pm$ sys. & HQLGT & {$[33]$} \\
\hline \hline
\end{tabular}


A recent Hamiltonian Monte Carlo study [28] of the flux tube model determined hybrid masses without using the questionable approximations of the earlier flux tube model studies, such as an adiabatic separation of quark and flux-tube motion and a small oscillation approximation for the flux tube. This Monte Carlo study generally confirmed the accuracy of the earlier flux-tube model mass estimates, both for $q \vec{q}$ and $c \vec{c}$ mesons (compared to experiment) and for hybrids (compared to the earlier approximate analytical calculations). These flux tube predictions are shown in Fig.1 below for light quarks and in Fig. 2 in the discussion of charmonium hybrids.

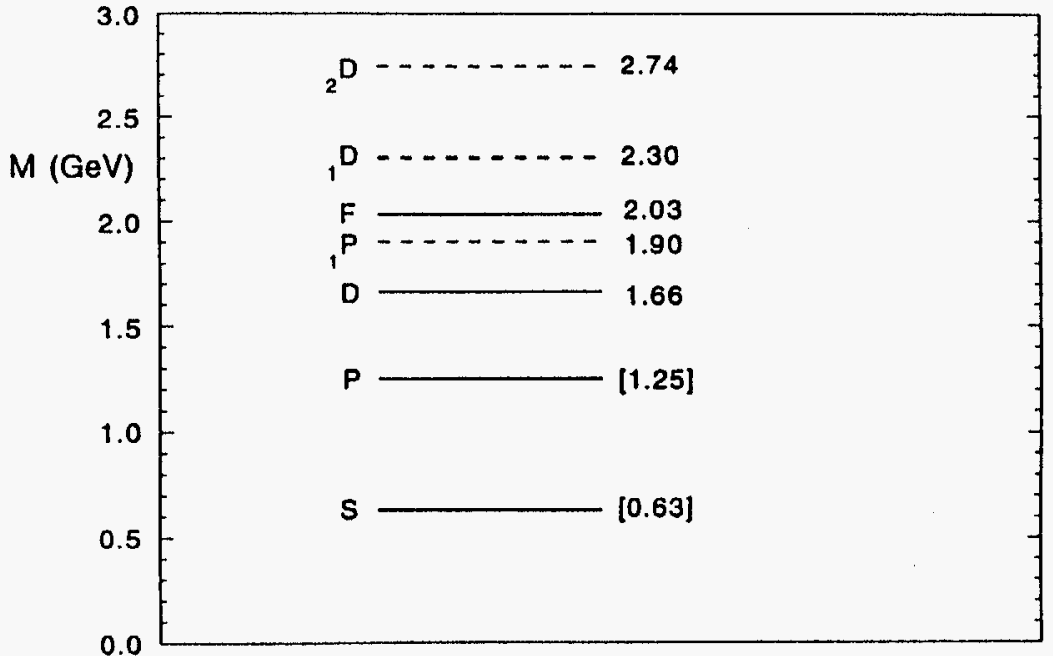

Fig.1. The light qqbar $(q=u, d)$ and hybrid spectrum in the flux tube model [28].

By varying the model parameters over a plausible range, this study concluded that the lightest hybrid masses in the flux tube model were

$$
M\left(H_{u, d}\right)=1.8-1.9 \mathrm{GeV}
$$

for light quark hybrids and

$$
M\left(H_{c}\right)=4.1-4.2 \mathrm{GeV}
$$

for charmonium hybrids. Excited hybrids were also considered, and the first hybrid orbital excitation $\left({ }_{\Lambda} L={ }_{1} D\right)$ was found at about $2.3 \mathrm{GeV}, 400 \mathrm{MeV}$ above the lightest $\left({ }_{1} P\right)$ hybrids. The same numerical result was found earlic by Merlin [26] using the adiabatic approximation. 'This , $D$ multiplet conliains the $J^{P C}$ states $(1,2,3)^{ \pm \mp}$ and $2^{ \pm \pm}$, which includes the exotics $1^{-+}, 2^{+-}$and $3^{-+}$. One way to test the experimental candidates for ground-state bybrirl: near $1.8 \mathrm{GeV}[40]$ and 1.6-2.2 GeV [41] would be to search for member's of thi. exciled,$D$ hybrid multiplet about $0.4 \mathrm{GeV}$ higher in mass.

\section{Light hybrid decay modes.}

Theoretical models predict rather characteristic two-body decay modes for hybrids. Both constituent gluon [20] and fux tube [27] models find that the lightest hybrids decay preferentially to pairs of one $L_{q \bar{q}}=0$ and one $L_{z i j}=1$ meson "S+P", for example $\pi \int_{1}$ and $\pi b_{1}$. These unusual modes previousl received little experimental attention because they involve complicaled fina states, which may explain why hybrids were not discovered previously. The flux-tube decay predictions of Isgur, Kokoski and Paton [27] are quite iuler esting because they suggest that many hybrids are so broad that they will be effectively invisible, whereas a few hybrids should be narrow enongh to be as ily observable in certain channels. The $I=1 . J^{r} C=1^{-+}$exotic had alsarty been cited as an attractive experimental candidate, and this work suggesterl that this state should be relatively narrow, $\Gamma_{t o t} \approx 200 \mathrm{MeV}$, and that. the $\mathrm{S}+\mathrm{P}$ modes $\pi b_{1}$ and $\pi f_{1}$ should be the dominant final states. These studies have motivated several experimental investigations of $\pi b_{1}$ and $\pi f_{1}$, which show possible indications of resonant amplitudes in $1^{-+}$

These original flux tube decay calculations were for the three exotic $f^{\text {Pr }}$ quantum numbers in the lowest flux-tube multiplet. Since this multiplet ron tains a total of eight $J^{P C}$ assignments, $1^{ \pm \pm}$(for $\left.S_{q \bar{q}}=0\right)$ and $2^{ \pm \mp} ; 1^{ \pm \mp} ; 0^{ \pm \mp}$ (for $S_{q \bar{q}}=1$ ), one might wonder whether any of the nonerotic hybricts are narrow enough to be observed. The decay amplitudes of these nonexolic hybrids were recently calculated by Close and Page [29], who also checked the exotic decay amplitudes and found reasonable numerical agreement with Isgur, Kokoski and Paton.

Close and Page predict that many of these nonexotic hybrids are also so broad as to be effectively unobservable. There are two striking exceptions. One is a $1^{--} \omega$-hybrid with a total width of only $\approx 100 \mathrm{MeV}$, which decays 10 $K_{1}(1270) K$ and $K_{1}(1400) K$; this should be searched for in $K_{1} K$ final states. perhaps in photoproduction. $\Lambda$ second interesting nonexolic hybrid is a $\pi_{2}$, with $\mathrm{r}_{\text {tot }} \approx 170 \mathrm{MeV}$. This may be the high-mass stale which has becn reported in several photoproduction experiments a mass near $1775 \mathrm{MeV}$ [40]. (O).her notable conclusions are that 1) several other hybrids, including exolics, haw total widths near $300 \mathrm{MeV}$ and so should be observable, and 2) the $l=00^{+-}$ 
exotic found by Isgur et al. to have $\Gamma_{b_{1} \pi}=250 \mathrm{MeV}$ actually has very large $K_{1} K$ modes and so should be unobservable.

In addition Close and Page investigate the "forbidden" decay modes such as $\hat{\rho}(1900) \rightarrow \rho \pi$, and find that, due to differences in the $\rho$ and $\pi$ spatial wavefunctions, these $\mathrm{S}+\mathrm{S}$ modes are present with partial widths of typically $\sim 10 \mathrm{MeV}$. An important $\rho \pi$ coupling was found earlier by deViron and Govaerts [38] using QCD sum rules. Thus it is interesting to search relatively straightforward modes such as $\rho \pi$ for hybrids, in addition to the favored but more difficult $\mathrm{S}+\mathrm{P}$ modes such as $b_{1} \pi, \pi f_{1}$ and $K_{1} K$

\section{Prospects for charmonium hybrids at a TCF.}

The predictions of the recent flux tube model calculations ( [28], shown below) and heavy-quark LGT [33] that hybrid charmonium states should appear beginning at 4.1-4.2 GeV are especially relevant for the physics program of a Thu: Oharm Fhetary,

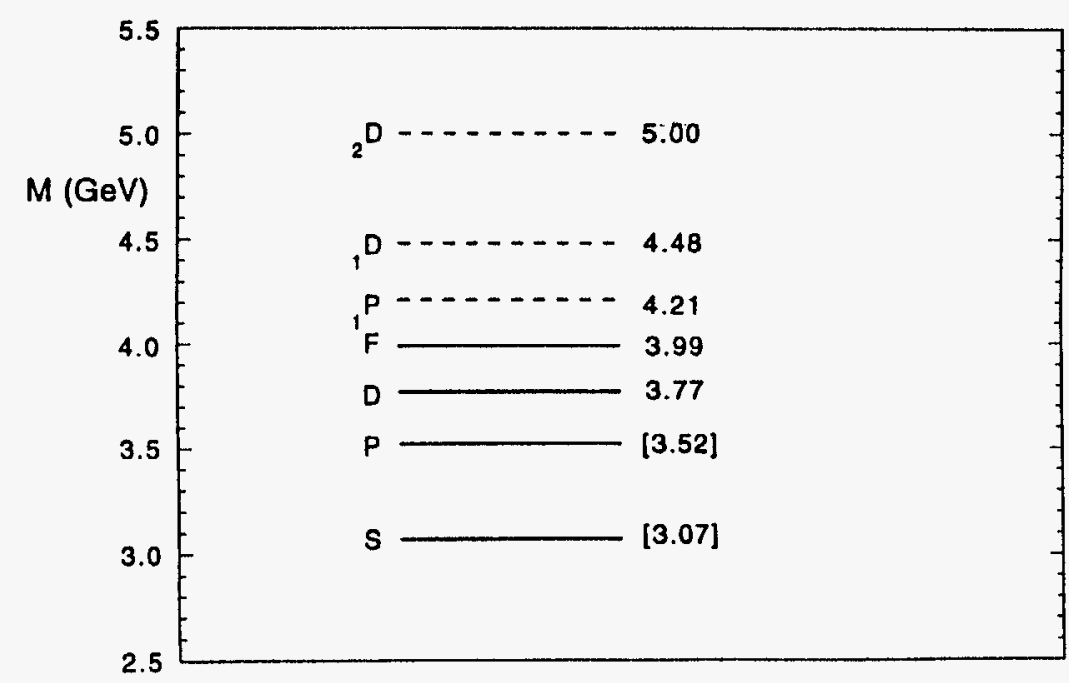

Fig.2. Charmonium and ccbar-hybrid masses in the flux tube model [28].

Charmonium spectroscopy is rather well understood up to about $3.8 \mathrm{GeV}$, so searches for unusual states should be straightforward near this mass. Since only a few open charm channels occur below $4.3 \mathrm{GeV}$, for a considerable range of masses one might anticipate rather narrow hybrid resonances. This pos- sibility is supported by the theoretical preference of hybrids for $\mathrm{S}+\mathrm{P}$ decis modes, which have thresholds of about $4.3 \mathrm{GeV}$ for $c \bar{c}$ and 11.0 (GeV for bit Calculations of the decay widths of charmonium hybrids have been carricd in the flux tube model by Close and Page [31], assuming masses of $\approx 4.1 .1$. $\mathrm{GeV}$. The partial widths (to $D^{*} D$ ) are found to be quite small, typically onl $\sim 1-10 \mathrm{MeV}$. Thus if there are relatively unmixed charmonium hybricls, the $1^{--}$vector hybrids should appear as narrow spikes in $R$ in this mass range For this reason a detailed scan of $R$ starting near the open charm threshol, would be a first priority at a Tau-Charm Factory.

Close and Page subsequently speculate about a more complicated possibil ity, which is that the $\psi(4040)$ and $\psi(4160)$ may be equal-weight linear com binations of $3 S|c \bar{c}\rangle$ and $1^{--} c \bar{c}$-hybrid basis states. (The usual assignment is that the $\psi(4040)$ is a $3 S c \bar{c}$ and the $\psi(4160)$ is a $2 D c \bar{c}[43]$.) The Close-Pige linear combinations would explain why the $e^{+} e^{-}$widths are approximatch equal and relatively large for both states, which is surprising if one is a 1) wave $c \tilde{c}$. The assignments for the $\psi$ states above open-charm thresholds (an be tested by measurements of their branching fraetlons to $\left.\left.D D, D^{*} 1, \ldots, 1\right)_{s}^{*} 1\right)^{*}$ The branching fractions predicted by these models are very scnsitive to $1 \mathrm{ll}$. initial state assignments [42]; unfortunately they have not yet been measured accurately. Determination of these branching fractions would be another light priority at a TCF.

Finally, we note that the non-vector hybrids can also be produced al in TCF through a "continuum cascade", as suggested by D.Bugg, and discussid in references $[43,44]$. In this approach one produces a high-mass $c \bar{c}$ system in the continuum, for example at $5 \mathrm{GeV}$; this may then decay hadronically to hybrid charmonium levels of various $J^{P C}$ accompanied by a light haclrou o. hadrons. The $c \bar{c}$-hybrid in turn decays hadronically to a characteristic stalc such as the $\psi$. Thus one can search for example for the decay chain

$$
e^{+} e^{-} \rightarrow c \bar{c} \rightarrow H_{c} \eta ; \quad H_{c} \rightarrow \eta \psi ; \quad \psi \rightarrow e^{+} e^{-}
$$

in the final state $\eta \eta e^{+} e^{-}$, triggering on a lepton pair at the $\psi$ mass and $\gamma \gamma$ pair from the two $\eta$ s. The $\eta \psi$ invariant mass distribution can then be studied fou evidence of hybrids or $c \bar{c}$ states. Other quantum numbers can be inwestigallacl by replacing $\eta$ by other hadrons, for example $(\pi \pi)_{S}$, in the hadronic cascarles

\section{E. Hybrid Experimental Candidates}

There are several experimental candidates for hybrids, but just as for glu balls there are no generally accepted states at present.

In the exotic channels (which would provide the most convincing evideme. for hybrids), previous claims by GAMS that a resonant signal had been d. 
tected in the $1^{-+}$wave of $\pi \eta[45]$ have now been withdrawn. A KEK experiment [46] finds evidence for a resonant $1^{-+} \pi \eta$ wave, but with the mass and width of the $a_{2}(1320)$; this surprising result obviously must be checked carefully for "feedthrough" of the $a_{2}$ amplitude. VES [47] has studied $\pi \eta$ and $\pi \eta^{\prime}$ and report a broad, higher-mass effect in $\pi \eta$ and especially in $\pi \eta^{\prime}$, near 1.6 $\mathrm{GeV}$. The phase motion of the $1^{-+}$component has not yet been determined. Studies of the $\pi f_{1}$ final state suggested by the flux tube model are underway $[41,47]$, and preliminary evidence for a possible $1^{-+}$signal has been reported by $\mathrm{E} 818$ at BNL [41].

There have been several observations of a photoproduced $I=1$ state in $\rho \pi$ and $\pi f_{2}$ at about $1775 \mathrm{MeV}$ [40], which is too heavy to be the $\pi_{2}(1670)$ without complicated interference effects. Although the quantum numbers of this state have not been determined definitively, $1^{-+}$is preferred over $2^{-+}$. A possible narrow $1^{-+}$state has been reported by GAMS in $\eta \eta^{\prime}$ at a mass of $1910 \mathrm{MeV}$ [48]; here there are rather few events, so it will be important to improve the statistics. Several experiments plan future studies of these channels, including E818 (to study $\left.\pi^{-} f_{1}\right)[49]$ and E852 (to study $\pi f_{1}$ and $\pi \eta$ ) [50] at I3NL.

In addition to exotic hybrids there are several nonexotic candidates; recall for example the Close-Page result that a hybrid with $\pi_{2}$ quantum numbers is expected to be relatively narrow, and should be visible in $\pi f_{2}$. One way to distinguish hybrids from $q \vec{q}$ spin-singlet states is through their strong decay amplitudes; for example, in the $\pi_{2}$ sector the relative $F / P$ and $D / S$ amplitude ratios in $\pi_{2}(q \vec{q}) \rightarrow \rho \pi$ and $\pi f_{2}$ are reasonably well constrained in the ${ }^{3} P_{0}$ and flux tube decay models [51]. These decay models provide an interesting selection rule for $q \bar{q}$ decays; they forbid the decay of a spin-singlet $q \bar{q}$ state to two final spin-singlet quarkonia,

$$
(q \vec{q})_{S=0} \nrightarrow(q \vec{q})_{S=0}+(q \vec{q})_{S=0} .
$$

III the $\pi_{2}$ channel this selection rule forbids the decay of a ${ }^{1} D_{2} q \bar{q} \pi_{2}$ to a ${ }^{1} S_{0}$ $\pi$ plus a ${ }^{1} P_{1} b_{1}$,

$$
\pi_{2}(q \bar{q}) \nrightarrow \pi b_{1}
$$

but allows it for a hybrid $\pi_{2}$ which does not have the $q \ddot{q}$ pair in an $S=0$ conliguration. (Close and Page find the $\pi b_{1}$ mode of $a \pi_{2}$ hybrid should be rather large, so it is especially important to search the $\pi b_{1}$ channel for evidence of a $2^{-+}$signal.

Other nonexotic hybrid candidates which have been suggested recently are a. $\pi(1800)$ reported by VES $[52]$ and the nonstrange $1^{--}$states near 1.4-1.7 GeV [53]. The $\pi(1800)$ is cited as a possible hybrid because it has unusual branching fractions, including a significant coupling to $\pi \eta \eta$, apparently through the glucball candidate $f_{0}(1500) \rightarrow \eta \eta$. This $\pi(1800)$ is also reported by VES in $\omega \rho$, $\eta a_{0}(980), \pi f_{0}(980)$ and $\pi f_{0}(1300)$. The decay mode $\pi(1800) \rightarrow \rho \pi$ is notally absent, and $\pi f_{2}$ is also weak or absent.

Although the weakness of the $\rho \pi \mathrm{S}+\mathrm{S}$ mode is indeed suggestive of a 11 brid, a $\pi(1800)$ second radial excitation is expected in quark potential models (Godfrey and lsgur [54] predict $1.88 \mathrm{GeV}$ ), so one should consider this assignment as well. Radial quarkonia can have unusual branching fractions du to nodes in their decay amplitudes, and in the ${ }^{3} P_{0}$ decay model with sllo wavefunctions the amplitude for $\pi(3 S) \rightarrow \rho \pi$ has a node at $M=1.88$ (in for $\beta=0.35 \mathrm{GeV}$. The weakness of the $\rho \pi$ mode is therefore understandiable for a $3 S$ state. The same model however predicts a weak $\pi f_{0}(1: 300)$ morle. which disagrees with experiment. 'The decay amplitude for $\pi(3 S) \rightarrow \pi \rho(2,5)$ is predicted to be quite large [55], so a search for a $\pi \rho(1450)$ final state would be useful.

The unusual properties of the nonstrange $I=0$ and $I=1$ vectors near $1.5 \mathrm{GeV}$ have led to suggestions that hybrid vector states may be present near this mass $[53,56]$. In $I=1$, for example, the two states $p(1450)$ and $\rho(1700)$ are usually assigned to $2^{3} S_{1}$ and ${ }^{3} D_{1}$ respectively, but the very latger $\rho(1450) \rightarrow 2\left(\pi^{+} \pi^{-}\right)$mode [56] is in conflict with quark model expectation: for a $2^{3} S_{1}$ state [56-58]. A better understanding of these vector states ma! require a detailed isobar analysis of their quasi two-body strong decay morles.

These comparisons of strong decay modes illustrate the importance of hav ing an accurate understanding of the decays of madially excited $q \bar{q}$ states. ('are ful studies of the strong decays of radially excited $q \bar{q}$ candidates such as the $\pi(1300), \rho(1450), \phi(1680), \pi(1800)$ and so forth will be required if we are to distinguish $q \bar{q}$ from non- $q \bar{q}$ states with identical quantum numbers.

\section{MULTIQUARK SYSTEMS AND MOLECULES}

\section{A. Introduction}

Multiquark systems have had a complicated history, and current hlecorelinal expectations for these states now differ radically from the earliest suggestions. In the pre-QCD quark model era it was thought that multiquark hadrons: should exist as resonances in the hadron spectrum. After the discovery of QCD and confinement it was still widely expected that multiguark liadrons: should exist (in color singlet sectors), and models typically predicted a very rich spectrum of states. In the light $q^{2} \bar{q}^{2}$ sector these "baryonium" resonances wole expected to appear beginning at about $1 \mathrm{GeV}$. It was clear lowerer that llwe. were problems with these predictions, because in the relatively uncomplicallal navor-exotic channels such as $I=2, J^{P C}=0^{++}$no $q^{2} \bar{q}^{2}$ resonances wer. observed [59] whereas they were predicted to be relatively light $(\approx 1.2$ (ic) in the MIT bag model). Similarly, the evidence for dilambda hypermuclei f(it) 
makes the existence of an $H$ six-quark resonance well below $\Lambda \Lambda$ threshold (another bag model prediction) appear very unlikely.

The problem with these predictions of multiquark resonances such as $q^{2} \bar{q}^{2}$ was that they were above $(q \bar{q})(q \vec{q})$ thresholds, and could spontaneously dissociate "fall-apart" into two mesons [61]. Thus the mass predictions in models which assumed a priori that the $q^{2} \bar{q}^{2}$ system existed as a single hadron were spurious, because the physical eigenstates were usually a continuum of scattering states [62]. Whether single multiquark clusters exist as resonances under any conditions is a detailed dynamical question, which should be investigated using models that allow the system itself freedom to choose between a single cluster or separate color singlets. At present it appears that single $q^{2} \tilde{q}^{2}$ hadronic clusters may only exist as resonances in heavy-light systems such as $c^{2} \tilde{q}^{2}\{63\}$.

More realistic models of multiquark systems were subsequently developed which gave the $q^{2} \vec{q}^{2}$ system freedom to choose dynamically between a bound system and a two-meson scattering state. The variational calculations of Weinstein and Isgur [64] are the best known of these studies; in this work it was found that most $0^{+}$sectors of the light $q^{2} \tilde{q}^{2}$ system had two free mesons as the ground state, but that the $I=0$ and $I=1 q s \bar{q} \bar{s}$ sectors actually had a weakly bound, deuteronlike $K \bar{K}$ pair as the ground state. These states were obvious assignments for the problematical $f_{0}(980)$ and $a_{0}(980)$ resonances, which were difficult to explain as ${ }^{3} P_{0} q \bar{q}$ states but could easily be understood as $K \tilde{K}$ systems with nuclear binding energies of $10 \mathrm{~s}$ of $\mathrm{MeV}$. These states have been the "prototypes" for hadron molecules, although they remain somewhat controversial. We note in passing that molecule states as a general category are not at all controversial, since the $>10^{4}$ known nuclear levels are all examples of hadronic molecules. Here we will discuss meson molecules; candidates also exist in baryon sectors, for example the $\Lambda(1405)$, which may be a $\bar{K} N$ bound system [65].

Signatures for the a priori most likely molecular states [66] can be abstracted from our experience with short-ranged hadronic forces and the Weinstein-Isgur results:

1) $J^{P C}$ and flavor quantum numbers of an $L=0$ hadron pair.

2) A binding energy of at most about $50-100 \mathrm{MeV}$.

3) Strong couplings to constituent channels.

4) Anomalous EM couplings relative to expectations for conventional quark model states.

\section{B. Experimental molecule candidates}

1) $f_{0}(975)$ and $a_{0}(980):$ The " $K \bar{K}$-molecules".

Weinstein and Isgur [64] found an exception to the fall-apart phenomenom in the scalar sector, with parameters corresponding to the $q s \tilde{q} . \bar{s}$ system. Her weakly-bound deuteronlike states of kaon and antikaon were found to bo $1 \mathrm{l}_{\mathrm{w}}$ ground states of the four-quark system; Weinstein and Isgur refer to these as

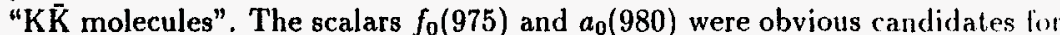
these states, having masses just below $K \bar{K}$ threshold and strong couplings 10 strange final states. Subsequently the $\gamma \gamma$ couplings of the $f_{0}(975)$ and $a_{0}(98(1)$ were found to be anomalously small relative to expectations for light ${ }^{3} /{ }^{\prime} 14$ states $(q=u, d)$, as discussed in references $[67,68]$. The status of the $K h$ molecule assignment and the many points of evidence in its favor have berll discussed recently by Weinstein and Isgur $[69,70]$.

Morgan and Pennington have argued against a molecule interpretation of the $f_{0}(975)$ [71]. Their criticism however applies to a $K \bar{K}$ potential model in which the $f_{0}(975)$ is a single pole in the scattering amplitude. The mor recent work of Weinstein and Isgur $[69,70]$ incorporates couplings to mescm meson channels and heavier ${ }^{3} P_{0} q \bar{q}$ states, so the physical resonances are not only $\mid K \bar{K})$. Since there has been much criticism of the idea of a pure $k h$ bound state, a direct quote from Weinstein and Isgur [69] (regarding the $I=\ldots 0$ state) is appropriate:

"Despite its name and location, the "KK $\bar{K}$ molecule" is not a simple $I K$ bound state. Its stability is dependent on its couplings to the other $I=1$ channels and at $E=M_{S}$. the coupled-channel wavefunction has substantin! components of the other atates."

Although the $f_{0}$ and $a_{0}$ states remain dominantly $K \bar{K}$, these modification: may answer the objections of Morgan and Pennington. Pennington suggest. that the term "deuteronlike" may be a misnomer, if couplings to other statc: than $K \bar{K}$ play an important rôle in these states [67]. Thus it appears that the important question regarding the $f_{0}$ and $a_{0}$ may be one of detail, specifically how large the subdominant non- $K \bar{K}$ components are in these states and how they can be observed experimentally.

The experimental measurements which would be most useful for sturlies of these states at a TCF are 1) their $\gamma \gamma$ widths, which are as yet rather poorly known, and 2) their cross sections in $\psi$ hadronic decays, in $\psi \rightarrow \omega / f_{11}$ and $\phi f_{0}$. (The latter are flavor-tagging and in studies at BES have shown that the $f_{0}(980)$ does appear to be a mixed flavor state.) Other intercsting measurements at low energies are the radiative transitions $\phi \rightarrow \gamma f_{0}$ and $\gamma\left(a_{1}\right.$. 
Which depend strongly on the scalar assignment [72] and may be measured at 1) $\backslash$ PHINE [73] and CEBAF [74].

\section{2) $f_{1}(1420)$}

Since the $f_{1}(1420)$ is above the $K^{*} K$ threshold of $1390 \mathrm{MeV}$ it is a candidate for a nonresonant threshold enhancement $\left(K^{*} \bar{K}^{\prime}+h . c.\right)$ rather than a molecular bound state. This possibility was suggested by Caldwell [75], and satisfies the criteria of lying just above the $K^{*} K$ threshold (antiparticle labels are implicit) and having quantum numbers allowed tor that pair in S-wave. The apparent width of the enhancement should not be narrower than the intringic wiclth of the $K^{\circ}$, and indeed the PDG values are similar, $\Gamma\left(f_{1}(1420)\right)=56 \pm 3 \mathrm{MeV}$ and $\Gamma\left(K^{*}\right)=50 \mathrm{MeV}$. Longacre $[76]$ found that a morlel with an S-wave nonresonant $\left(K^{*} \bar{K}+h . c\right.$.) enhancement gives a good description of this state, and Isgur, Swanson and Weinstein [77] also favor this possibility. The (offshell) $\gamma \gamma^{*}$ couplings of the $f_{1}(1420)$ relative to expectations for a $1^{\text {t+ }} s \bar{s}$ state may provide a test of the hadron-pair model.

$\Lambda$ nother test of this $K^{*} K$-assignment is in radiative transitions; the dominant radiative mode of a $K^{*} K$ system will arise from the radiative transition of the $K^{*}$ constituent, $K^{*} \rightarrow K \gamma$, implying a partial width of

$$
\mathrm{I}\left(f_{1}\left(K^{*} K\right) \rightarrow \gamma K \vec{K}\right) \approx 80 \mathrm{KeV},
$$

and a characteristic pattern of preference for $K^{\circ} K^{\circ}$ over $K^{+} K^{-}$by about a factor of two. An $s \bar{s}$ state would give a similar radiative partial width, $\mathrm{I}\left(f_{1}(1420)(s \bar{s}) \rightarrow \gamma \phi\right) \approx 50 \mathrm{KeV}$ if we scale from the $\Gamma\left(f_{2}^{\prime} \rightarrow \gamma \phi\right)=96 \mathrm{KeV}$ of Godfrey and lsgur [54]. Although the radiative rates are similar, there is a crucial difference in the two assignments: The $s \bar{s}$ decay is to $\gamma \phi$, so the final $K \bar{K}$ pair will clearly originate from a $\phi(1019)$ peak. The $K \bar{K}$ events from a $K * K$ system should instead have a broad distribution in invariant inass. Thus, the two $f_{1}(1420)$ ansignments can ensily be distinguiahed through the $\kappa K$ invariant mass distribution observed in $f_{1}(1420) \rightarrow \gamma K K$.

3) Other possible molecules.

There are many other possible molecular states, which can only be mentioned briefly here. In the meson sector these include the $f_{0}(1710)$, which could be a vector-vector molecule involving $K^{*} K^{*}[78-80]$. This can be tested at a ' TCF' by searches for a large $K \bar{K} \pi \pi$ mode. Similarly, the $f_{0}(1500)$ glueball candidate might be a nonstrange vector-vector system $[78,81]$, which would explain the weakness of the $K K$ mode. The $f_{0}(1365)$ should also be considered a possible vector-vector molecule, in view of its very large coupling to $\rho \rho$ despite the near absence of phase space. The $2^{++}$state reported by VES $[52]$ in $\rho^{o} \rho^{o}$ is another possible vector-vector molecule, although its appearance in the $\rho \rho$
D-wave may be a problem. The $\psi(4040)$, which shows a strong preforence for $D \bar{D}$ over $D^{*} \bar{D}^{*}$ (opposite to expectations from phase space) was onc of the earliest molecule candidates [82]. As the $c \bar{c}$ assignment for this state is a 3. radial excitation, this anomalous branching fraction may be due to a node in the decay amplitude near the $D^{*} \bar{D}^{*}$ momentum [42]. Finally, there are sercial molecule candidates in baryon sectors, such as the $\Lambda(1405)[65]$ (which as : possible $\bar{K} N$ is the earliest molecule candidate excluding nuclei), $K N$-flawn " $Z$ " exotics (discussed in $[83,84]$ ) and dibaryons [85].

Since molecular bound states are a special aspect of $2 \rightarrow 2$ hadroll swil tering amplitudes, one might anticipate that an understanding of these scint tering amplitudes will lead to reliable predictions of molecules. Throreticinl work along these lines is in progress; at present there are different. predictions for molecules depending on the scattering mechanism assumed. In one pion exchange models $[78,80]$ many bound states are predicted which slould be experimentally observable. In scattering calculations assuming quark-gluon forces (see $[79,83,86-89]$ and references cited therein) few channels arc found to have sufficiently strong attractions to form bound states; the vector-vector system [79,87] is one of the few. One of the principal linitations of harlion hadron scattering calculations at the quark-gluon level is the absence of "y" annihilation in most studies. Annihilation is known to be an inportant cllere when allowed, for example in the $K \bar{K}$ molecules $[69,70]$. An extension of thi work to include $q \vec{q}$ annihilation is in progress $[90]$.

\section{ACKNOWLEDGMENTS}

I would like to thank the organisers of the Argonne Cau-Cham morling especially Josê Repond and Jasper Kirkby, for the opportunity to prescell these results and to discuss hadron physics with my follow participanls. I inn

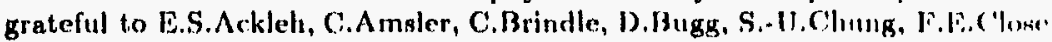
G.Condo, K.Danyo, A.Donnachie, A.Dzierba, P.Geiger, S.Godfrey, N.Isign. P.R.Page, M.R.Pennington, E.S.Swanson and N.Tönquist for discussions am material that contributed to this work. This research was sponsored in part lin the United States Department of Fnergy under contract DE-A(05-8401221400. managed by Lockheed Martin Energy Systems, Inc.

(1) F. L.C. lose, Rep. Prog. Phys. 51, 83:3 (1988).

[2] For the earlier LGT glueball literature see for example C.Michard and M.'Teper. Nucl. Phys. B314, 347 (1989); P.deForcrand, G.Schierholz, H.Schucider inul 
M.Teper, Phys. Lett. B152, 107 (1985); G.Berg, Nucl. Phys. B221, 109 (1983); and references cited therein.

[3] G.Bali et al, Phys. Lett. B309, 378 (1993).

(4) H.Chen et al., IBM report IBM-HET-94-1 (contribution of A. Vaccarino to Lattice 93).

(5) J. Sexton et al., IBM report IBM-HET-94-5 (contribution to I,attice 94)

[6] C.Amsler and F.E.Close, "Evidence for Glueballs", Rutherford Laboratory and CERN report CCL-TR-95-003 (April 1995); ibid., Rutherford Laboratory reports RAL-95-036 (May 1995) and RAL-TR-95-003 (July 1995).

[7] E.S.Ackleh, T.Barnes and E.S.Swanson, Oak Ridge National Laboratory report ORNL-CTP-95-09.

[8] See for example E.S.Ackleh and T.Barnes, Phys. Rev. D45, 232 (1992); T.Barnes, in Proceedings of the IXth International Workshop on PhotonPhoton Collisions (World Scientific, 1992) eds. D.O.Caldwell and II.P.Paar, pp.263-274; and references cited therein.

[9] J.K.Bienlein (Crystal Ball Collaboration), in Proceedings of the Ninth International Workshop on Photon-Photon Collisions (La Jolla, 22-26 March 1992), eds. D.O.Caldwell and H.P.Paar (World Scientific, 1992), pp.241-257.

[10] V.V.Anisovich et al., Phys. Lett. B323, 233 (1994); C.Ansler, in Proceedings of the XXVII Int. Conf. on High Energy Physics (Glasgow, 20-27 July 1994) Zürich report UZH-PH-50/94.

[11] C.Amsler, personal communication.

[12] D.Bugg et al., Phys. Lett. B353, 378 (1995).

[13] R.M. Baltrusaitis et al., Phys. Rev. Lett. 56, 107 (1986).

[14] T.Huang, contribution to the Argonne Workshop on a Tau-Charm Factory (June 1995); see also T.Huang et al., CCAST report BIHEP-TH-95-11.

[15] S.Godfrey, R.Kokoski and N.Isgur, Phys. Jett. B141, 439 (1984).

[16] S.Godfrey, personal communication; II.G.Blundell and S.Godfrey, "The $\xi(2220)$ Revisited: Strong Decays of the $1^{3} F_{2}$ and $1^{3} F_{4} s \bar{s}$ Mesons.", Carleton University report OCIP/C 95-11.

[17] D.Horn and J.Mandula, Phys. Rev. D17, 898 (1978).

[18] T.Barnes, "The Bag Model and Hybrid Mesons", in Proceedings of the SIN Spring School on Strong Interactions (Zuoz, Switzerland, April 9-17, 1985). $\Lambda$ lso distributed as University of Toronto report UTTPT-85-21 ( $\Lambda$ pril 1985).

[19] T.Barnes, Caltech Ph.D. thesis (1977), unpublished; T.Barnes, Nucl. Phys. B158, 171 (1979); 'T.Barnes and F.E.Close, Phys. Lett. 1163, 365 (1982); M.Chanowitz, and S.R.Sharpe, Nucl. Phys. I3222, 211 (1983); T.Barnes, F.E.Close and F.deViron, Nucl. Phys. B224, 241 (1983); M.Flensburg, C.Peterson and L.Sköld, Z. Phys. C22, 293 (1984).

[20] M.Tanimoto, Phys. Lett. 116B, 198 (1982); Phys. Rev. D27, 2648 (1983); A.LeYaouane, L.Oliver, O.Pène, J.C. Kaynal and S.Ono, Z. Phıs. (:28, 309 (1985); F.Iddir, A.LeYaonanc, L.Oliver, O.Pène, J.-C.Raynal and S.Ono, Phys.
Lett. B205, 564 (1988); S.Ishida, II.Sawazaki, M.Oda and Ki Launda W Rev. D47, 179 (1992); Prog. Theor. P'lys. 82, 119 (1989).

[21] J.M.Cornwall and S.F.Tuan, Phys. Lett. B136, 110 (1984)

[22] N.Isgur and J.Paton, Phys. Lett. 124B, 247 (1983).

[23] J.Merlin and J.Paton, J. Phys. G11, 439 (1985).

[24] N.Isgur and J.Paton, Phys. Rev. D31, 2910 (1985).

[25] J.Merlin and J.Paton, Phys. Rev. D35, 1668 (1987).

[26] J.Merlin, Oxford University Ph.D. thesis (unpublished); J.Paton, personal inn. munication.

[27] N.Isgur, R.Kokoski and J.Paton, Phys. Rev. Lett. 54, 869 (1985).

[28] T.Barnes, F.E.Close and E.S.Swanson, Oak llidge National laboralow / Rutherford Laboratory Report ORNL-CTP-95-02 / R LL-94-106, IM. ph/9501405, Phys. Rev. D (to appear).

[29] F.E.Close and P.R.Page, Nucl. Phys. I3443, 233 (1995).

[30] F.E.Close and P.R.Page, Rutherford Laboratory report RAL-9.1-122. I I $\mathrm{ph} / 9412301$.

[31] F.E.Close and P.R.Page, Oxford University / Rutherford Laboratory mun OUTP-95-13P / RA1,-95-122, hep.ph/9507.407.

[32] P.Ilasenfratz, R.R.Ilorgan, J.Kuti and J-M.Richard, Phys. Let1. 9513. 2." $(1980)$.

[33] S.Perantonis and C.Michael, Nucl. Phys. B347, 854 (1990), and werences rite. therein.

[34] I.I.Balitsky, D.1.Dyakanov and A.V.Yung, Plyys. Let1. 112B, 71 (1982); Sor. Nucl. Phys. 35, 761 (1982); Z. Phys. C33, 265 (1986).

[35] J.I.Latorre, S.Narison, P.Pascual and R.Tarrach, Pliys. Lett. 1.1713, 16!) (19S1 J.I.Latorre, P.Pascual and S.Narison, Z. P'hys. C34, 347 (1987); S.Narisol "QCD Spectral Sum Rules", Lecture Notes in Pliysics Vol.26, p.375 ( Kioll Scientific, 1989).

[36] J.Govaerts, F.deViron, D.Gusbin and J.Weyers, Phys. I,ett. 1281\}, 262 (1!) : ! (E) Plyys. Lett. 136B, 445 (1983); J.Govaerts, L.J.Reinders, M.R.Hulinstcin an J.Weyers, Nucl. Phys. B258, 215 (1985); J.Govaerts, L.J.Reinders and J. Winer Nucl. Phys. B262, 575 (1985); J.Govaerts, L.J.Reinders, P.Francken, X.(im, and J.Weyers, Nucl. Phys. B284, 674 (1987).

[37] J.Govaerts, F.deViron, D.Gusbin and J.Weyers, Nucl. Pliys. 132.18, I (198 1).

[38] F.deViron and J.Govaerts, Phys. Rev. Lett. 53, 2207 (198.1).

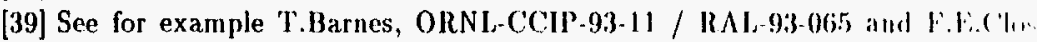
RAI-93-053, in Proceedings of the Third Workshop on the Tan-( Harm lachen (Marbella, Spain, 1-6 June 1993); 'T.Barnes, ORNL-CCIP-93-1.1 / RML, 93 wi in Proceedings of the Conference on Exclusive leactions at lligh Momenul Transfers (Marciana Marina, Elba, Italy, 24-26 June 1993); F'L.('lose. Rn! Prog. Phys. 51, 833 (1988); (..Dover, in Proceedings of the Second Birnmi: Conference on Low Energy Antiproton Physirs (Commayeur, 14-19 Sepr. 1!n? 
A.Dzierba, Indiana University report IUIILE-93-2, in Proccedings of Ilie BNI. meeting on Future Directions in Particle and Nuclear Pliysics at Multi-(jeV Iladron Facilities (Brookhaven, N.Y. 4-6 March 1993); S.Codfrey, in Proceedings of the BNL Workshop on Glueballs, Ilybrids and Exotic Hadrons ( $\Lambda \mathrm{IP}$, 1989), ed. S.-U. Chung; D.Hertzog, Nucl. P'hys. A558, 499c (1993); N.Isgur, (EBAF-TH1-92-31, in Proceedings of the XXVI International Conference on Iligh Energy Physics (Dallas, August 1992); G.Karl, Nucl. Phys. A558, 113c (1993).

[.10] G.Condo et al., Phys. Rev. D43, 2787 (1991); this state may have been seen earlier by D.Aston et al., Nucl. Phys. B189, 15 (1981).

[11] J.Il.Lee et al., Phys. Lett. H323, 227 (1994).

[42] P.R.Page, Nucl. Phys. B446, 189 (1995); see also A.LeYaouanc et al., Phys. Lett. B71, 397 (1977); ibid., Phys. Lett. B72, 57 (1977).

[43] T.Barnes, in Proceedings of the 3rd Workshop on the Tau Charm Factory (EdiLion Frontieres 1994), eds. J.Kirkby and R.Kirkby, p.11.

[14] F.E.Close, in Proceedings of the 3rd Workshop on the Tall Charm Factory (Edition Fronticres 1994), eds. J.Kirkby and R.Kirkby, p.73.

[15] D. Alde ot al., Phys. Lett. B205, 397 (1988).

[16] II. Aoyagi et al., Phys. Lett. B314, 216 (1993).

[17] G.M.Beladidze et al., Phys. Lett. B313, 276 (1993).

[48] Yu. Prokoshkin, presentation at IIADRON95.

[19] S.U.Chung, personal communication.

[50] A.Dzierba, personal communication.

[51] P.Geiger and F.S.Swanson, Phys. Rev. D50, 6855 (1991), find that $\pi_{2}(q \bar{q}) \rightarrow \rho \pi$ has $F^{\prime} / P \approx 0.7$. The process $\pi_{2}(q \bar{q}) \rightarrow \pi f_{2}$ (not discussed in that reference) lias $D / S \approx 0.2$ and $G / S \approx 0.01$. (P.Geiger, personal communication, and $T$.Barnes, unpublished.)

[52] D.Rybachikov, contribution to HADRON95.

[53] A.Donnachie and Yu. Kalashnikova, Z.Phys C59, 621 (1993).

[5.4] S.Godfrey and N.Isgur, Phys. Rev. L32, 189 (1985).

[55] T.Barnes and F.E.Close, in preparation.

[56] A.B.Clegg and A.Donnachie, Z. Phys. C62, 455 (1994).

[57] G. Busetto and L.Oliver, Z. Phys.C 20, 247 (1983).

[58] R.Kokoski and N.Isgur, Phys. Rev. D35, 907 (1987).

[59] W.Hoogland et al., Nucl. Phys. B126, 109 (1977).

[60) S.Aoki et al., Prog. Theor. Phys. 85, 1287 (1991), and references cited therein.

[61] R.J.Jaffe, Phys. Rev. Lett. 38, 195, 617E (1977).

[62] N.Isgur, Acta Physica Austraica, Suppl. XXVII, 177 (1985).

[63] J.P.Ader, J.M.Richard and P.Taxil, Phys. Rev. D25, 2370 (1982); G.Grondin, unpublished.

[6.1] J.Weinstein and N.Isgur, Phys. Rev. Lett. 48, 659 (1982); Phys. Rev. D27, 588 (1983); see also A.Astier et al., Phys. Lett. B25, 294 (1967); A.B.Wicklund et al., Phys. Rev. Lett. 45, 1469 (1980).

[65] R.If.Dalitz and S.F.Tian, Pliys. Rev. Loth. 2, 425 (1959); ibid., Mull. Pll! (NY) 3, 307 (1960); see also J.I.Sakurai, Aun. P’hys. (NY) II, 1 (19)(60).

[66] 'T.Barnes, "Signatures for Molecules", Invited contribution to the XXIX 12 . contres de Moriond, (Meribel, France, 19-26 March 1994); Oak Ridgo Natium.1 Laboratory report ORNI, CCIP/94-08; proceedings published as "(2) (1) allil High Energy Hadronic Interactions" (Editions Frontieres, (iif-sur-Yvette, I991). pp.587-598.

[67] M.R.Pennington, University of Durham report D'TP-94/26 ( $\Lambda$ pril 199.1). IP. ceedings of the Meeting on 'Two-Photon Physics from D $\Lambda \Phi N E$ to LWP'200 and Beyond, eds. F.Kapusta and J.Parisi (Paris, February 1994).

[68] 'T.Barnes, Phys. Lett. 165B, 434 (1985); E.P.Shabalit, Yad. Fi\% 46, 852 (198 T.N.Truong, in Proceedings of the $11 \mathrm{ADNON}$ '89 International Mecting lladron Spectroscopy (Ajaccio, 1989), pp.645; N.Brown and F.E.C.lose, 13ut lice ford Laboratory report RAL-01-085.

[69] J.Weinstein and N.Isgur, Phys. Rev. D41, 2236 (1990).

[70] J.Weinstein, Phys. Rev. D47, 911 (1993).

[71] K.L.A11, D.Morgan and M.R.Pennington, Phys. Rev. D35, 1(333 (19:3i): D.Morgan and M.R.Pennington, Phys. Lett. 25813, 4.4 (1991); ibid., Rutherford Laboratory report RAL-92-070 (December 1992).

[72] F.E.Close, N.Isgur and S.Kumano, Nucl. Phys. B389, 513 (1993).

[73] N.Brown and F.E.Close, "Scalar Mesons and Kaons in Phi Radiative Denen

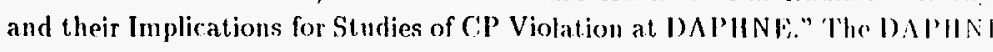
physics handbook, vol. 2, pp.447-463 (ed. L.Maiani), also distributed as Rullue ford Laboratory report 11 A I-91-085 (Der 1991).

[74] A.Dzierba et al., CEBAF proposal E-94-016; A.Dzierba, "Measuring Rare Ra diative Decays of the $\Phi$ Meson at CEBAF", in Procedings of the Secomd Wurk shop on Physics and Detectors for DAPHNE (DAPIIN L95).

[75] D.O.Caldwell, Mod. Phys. Lett. A2, 771 (1987); Procedings of the IBN1, Mirk shop on Glueballs, Ilybrids and Exotic Mesons (IPton, N.Y. 29 August - I September 1988), ed. S.U.Chung (AIP 1989), pp.465-471.

[76] R.S.Longacre, Phys. Rev. D42, 874 (1990).

[77] N.Isgur, E.S.Swanson and J.Weinstein, work in progress.

[78] N.Törnquist, in Procedings of the lntermational Conference on Iladrum Spectroscopy "IIADRON '91", (World Scientific, 1992; eds. S.Onedia anlel D.C.Peaslee), pp.795-798; Phys. Rev. Lett. 67, 556 (1991).

[79] K.Dooley, E.S.Swanson, and 'T.Barnes, Phys. Lett. 27513, 478 (1992); K.IDnole! in Proceedings of the 4th international Conference on Hadron Spectroscons "Hadron '91" (College Park, Md. 12-16 August 1991), (World Scientific, I!9!?!) pp.789-794.

[80] T.E.O.Ericson and G.Karl, Pliys. Lett. B309, 426 (1993); (i.Karl, Nucl. Plis A558, 113c (1993). 
[81] Yu.S.Kalashnikova, in Proceedings of the International Conference on Hadron Spectroscopy "HADRON '91", (World Scientific, 1992; eds. S.Oneda and D.C.Peaslee), pp.777-782.

[82] V.A.Novikov et al., Phys. Rep. C41, 1 (1978); M.B.Voloshin and L.B.Okun, JETP Lett. 23, 333 (1976); A.DeRújula, H.Georgi and S.L.Glashow, Phys. Rev. Lett. 38, 317 (1977); S.Iwao, Lett. Nuovo Cimento 28, 305 (1980).

[83] T.Barnes and E.S.Swanson, Phys. Rev. C49, 1166 (1994); see also K.Maltman and S.Godfrey, Nucl. Phys. A452, 669 (1986), who reach rather different conclusions regarding attractive channels.

[84] J.S.Hyslop, R.A.Arndt, L.D.Roper and R.L.Workman, Phys. Rev. D46, 961 (1992).

[85] R.A.Arndt, L.D.Roper, R.L.Workman and M.W.McNaughton, Phys. Rev. D45, 3995 (1992).

[86] T.Barnes and E.S.Swanson, Phys. Rev. D46, 131 (1992); for closely related work on meson-meson scattering see B.Masud, J.Paton, A.M.Green and G.Q.Liu, Nucl. Phys. A528, 477 (1991); D.Blaschke and G.Röpke, Phys. Lett. B299, 332 (1993); K. Martins, D. Blaschke and E. Quack, Phys. Rev. C51, 2723 (1995). A.LeYaouanc, L.Oliver, O.Péne and J.-C.Raynal, Phys. Rev. D42, 3123 (1990).

[87] E.S.Swanson, Ann. Phys. (NY) 220, 73 (1992).

[88] T.Barnes, E.S.Swanson and J.Weinstein, Phys. Rev. D46, 4868 (1992).

[89] T.Barnes, S.Capstick, M.D.Kovarik and E.S.Swanson, Phys. Rev. C48, 539 (1993).

[90] T.Barnes and E.S.Swanson, in preparation. 